\title{
Gravitational Charged Perfect Fluid Collapse in Friedmann Universe Models
}

\author{
M. Sharif *and G. Abbas ${ }^{\dagger}$ \\ Department of Mathematics, University of the Punjab, \\ Quaid-e-Azam Campus, Lahore-54590, Pakistan.
}

\begin{abstract}
This paper is devoted to study the gravitational charged perfect fluid collapse in the Friedmann universe models with cosmological constant. For this purpose, we assume that the electromagnetic field is so weak that it does not introduce any distortion into the geometry of the spacetime. The results obtained from the junction conditions between the Friedmann and the Reissner-Nordström de-Sitter spacetimes are used to solve the field equations. Further, the singularity structure and mass effects of the collapsing system on time difference between the formation of apparent horizons and singularity have been studied. This analysis provides the validity of Cosmic Censorship Hypothesis. It is found that the electric field affects the area of apparent horizons and their time of formation.
\end{abstract}

Keywords: Electric field; Gravitational collapse; Cosmological constant; Friedmann models.

PACS: 04.20.-q; 04.40.Dg; 97.10.CV

*msharif@math.pu.edu.pk

†abbasg91@yahoo.com 


\section{Introduction}

Gravitational collapse of a massive star is the result of its self gravity. It occurs when the internal nuclear fuel of the star fails to supply sufficiently high pressure to counter-balance gravity. Gravitational collapse is one of the most important problems in general relativity. According to the singularity theorems [1] there exist spacetime singularities in generic gravitational collapse. It has been an interesting problem to determine the nature of spacetime singularity. The cosmic censorship hypothesis $(\mathrm{CCH})$ [2] says that singularities appearing in gravitational collapse are always clothed by the event horizon.

The final fate of gravitational collapse of the massive star depends upon the choice of initial data and equation of state. Many efforts have been made to check its credibility but no final conclusion is drawn. For this purpose, Virbhadra et al. 3] introduced a new theoretical tool using the gravitational lensing phenomena. Also, Virbhadra and Ellis [4] studied the Schwarzschild black hole lensing and found that the relativistic images would confirm the Schwarzschild geometry close to the event horizon. The same authors [5] analyzed the gravitational lensing by a naked singularity and classified it as weak naked singularity and strong naked singularity. In a recent paper [6], Virbhadra used the gravitational lensing phenomena to find the improved form of the $\mathrm{CCH}$.

Oppenheimer and Snyder [7] studied dust collapse for the first time and showed that singularity is neither locally or globally naked. This means that they found black hole as a final fate of the dust collapse. Eardely and Smarr [8] found that inhomogeneous model undergoes to gravitational collapse by forming a singularity that can be either locally or globally naked.

There has been a growing interest to study gravitational collapse in the presence of perfect fluid and other general physical form of the fluid. Misner and Sharp [9] extended the pioneer work for the perfect fluid. Vaidya [10] and Santos [11] used the idea of outgoing radiation of the collapsing body and also included the dissipation in the source by allowing the radial heat flow. Markovic and Shapiro [12] generalized the pioneer work with positive cosmological constant. Lake [13] extended it for both positive and negative cosmological constant. Sharif and Ahmad [14]-[17] extended spherically symmetric gravitational collapse with positive cosmological constant for perfect fluid. The same authors [18] have also investigated plane symmetric gravitational collapse using junction conditions which has been extended to spherically symmetric gravitational collapse [19]. 
The behavior of electromagnetic field in strong gravitational field has been the subject of interest for the researchers over the past decades. According to Thirukkanesh and Maharaj [20], the inclusion of electromagnetic field in gravitational collapse predicts that the gravitational attraction is counterbalanced by the Coulomb repulsive force along with the pressure gradient. Sharma et al. 21] have concluded that electromagnetic field affects the value of red-shift, luminosity and mass of the relativistic compact objects. Nath et al. 222] have studied the gravitational collapse of non-viscous, heat conducting fluid in the presence of electromagnetic field. They concluded that electromagnetic field reduces pressure and favors the formation of naked singularity.

Recently, we have studied the effect of electromagnetic field on the gravitational collapse by taking spherically symmetric spacetime as interior region and Reissner-Nordström as exterior region of the star [23]. The present article investigates the previous work by taking the Friedmann universe models in the interior of star. In order to preserve the generic properties of the Friedmann universe models in the presence of electromagnetic field, we follow [24, 25] and assume that electromagnetic field is weak relative to matter, i.e., if $E^{2}$ is the electromagnetic field contribution in the system then $E^{2}<<\rho$. The main objectives of this work are the following:

- To study the physical interpretation of electromagnetic field and cosmological constant on gravitational collapse in the Friedmann universe models.

- To see the validity of $\mathrm{CCH}$ in this framework.

The plan of the paper is as follows: In the next section, the junction conditions are given. We discuss the solution of the Einstein-Maxwell field equations in section 3 . The apparent horizons and their physical significance are presented in section $\mathbf{4}$. Section 5 presents the singularity analysis. We conclude our discussion in the last section.

The geometrized units (i.e., the gravitational constant $G=1$ and speed of light in vacuum $c=1$ so that $M \equiv \frac{M G}{c^{2}}$ and $\left.\kappa \equiv \frac{8 \pi G}{c^{4}}=8 \pi\right)$ are used. All the Latin and Greek indices vary from 0 to 3, otherwise, it will be mentioned. 


\section{Junction Conditions}

We derive conditions for the smooth matching of two regions (interior and exterior of a star) on the surface of discontinuity. For this purpose, we assume that $\Sigma$ be a timelike $3 D$ hypersurface which divides two $4 D$ manifolds $V^{-}$ and $V^{+}$respectively. The interior manifold is taken as the Friedmann model

$$
d s_{-}^{2}=d t^{2}-a(t)^{2}\left[d \chi^{2}-f^{2}\left(d \theta^{2}+\sin \theta^{2} d \phi^{2}\right)\right],
$$

where $f_{k}(\chi)$ is defined as

$$
f(\chi)= \begin{cases}\sin \chi, & k=1 \\ \chi, & k=0 \\ \sinh \chi, & k=-1\end{cases}
$$

$k=1,0,-1$ correspond to closed, flat and open models respectively. $\chi(0 \leq$ $\chi<\infty$ for open and closed but $0 \leq \chi<\pi$ for flat) is the hyper-spherical angle and $a(t)$ is the scale factor. Further, $\chi$ is related to radial coordinate $r$ as follows: $r=\sin \chi$ (closed), $r=\chi$ (flat) and $r=\sinh \chi$ (open). The Reissner-Nordström de-Sitter spacetime is taken as the exterior manifold

$$
d s_{+}^{2}=Z d T^{2}-\frac{1}{Z} d R^{2}-R^{2}\left(d \theta^{2}+\sin \theta^{2} d \phi^{2}\right),
$$

where

$$
Z(R)=1-\frac{2 M}{R}+\frac{Q^{2}}{R^{2}}-\frac{\Lambda}{3} R^{2}
$$

$M$ and $\Lambda$ are constants and $Q$ is the charge.

The junction conditions are given as follows [26]:

1. The continuity of first fundamental form over $\Sigma$ gives

$$
\left(d s_{-}^{2}\right)_{\Sigma}=\left(d s_{+}^{2}\right)_{\Sigma}=d s_{\Sigma}^{2}
$$

2. The continuity of second fundamental form (extrinsic curvature) over $\Sigma$ yields

$$
\left[K_{i j}\right]=K_{i j}^{+}-K_{i j}^{-}=0, \quad(i, j=0,2,3)
$$

where $K_{i j}$ is the extrinsic curvature defined as 


$$
K_{i j}^{ \pm}=-n_{\sigma}^{ \pm}\left(\frac{\partial^{2} x_{ \pm}^{\sigma}}{\partial \xi^{i} \partial \xi^{j}}+\Gamma_{\mu \nu}^{\sigma} \frac{\partial x_{ \pm}^{\mu} \partial x_{ \pm}^{\nu}}{\partial \xi^{i} \partial \xi^{j}}\right), \quad(\sigma, \mu, \nu=0,1,2,3) .
$$

Here $\xi^{0}=t, \xi^{2}=\theta, \xi^{3}=\phi$ are the corresponding parameters on $\Sigma, x_{ \pm}^{\sigma}$ stand for coordinates in $V^{ \pm}$, the Christoffel symbols $\Gamma_{\mu \nu}^{\sigma}$ are calculated from the interior or exterior spacetimes and $n_{\sigma}^{ \pm}$are the components of outward unit normals to $\Sigma$ in the coordinates $x_{ \pm}^{\sigma}$.

The equation of hypersurface in terms of interior spacetime $V^{-}$coordinates is

$$
f_{-}(\chi, t)=\chi-\chi_{\Sigma}=0
$$

where $\chi_{\Sigma}$ is a constant as $\Sigma$ is a comoving surface forming the boundary of interior matter. Also, the equation of hypersurface in terms of exterior spacetime $V^{+}$coordinates is given by

$$
f_{+}(R, T)=R-R_{\Sigma}(T)=0 .
$$

When we make use of Eq.(2.8) in Eq.(2.1), the metric on $\Sigma$ takes the form

$$
\left(d s_{-}^{2}\right)_{\Sigma}=d t^{2}-a(t)^{2} f\left(\chi_{\Sigma}\right)\left(d \theta^{2}+\sin \theta^{2} d \phi^{2}\right) .
$$

Also, Eqs.(2.9) and (2.3) yield

$$
\left(d s_{+}^{2}\right)_{\Sigma}=\left[Z\left(R_{\Sigma}\right)-\frac{1}{Z\left(R_{\Sigma}\right)}\left(\frac{d R_{\Sigma}}{d T}\right)^{2}\right] d T^{2}-R_{\Sigma}^{2}\left(d \theta^{2}+\sin \theta^{2} d \phi^{2}\right),
$$

where we assume that

$$
Z\left(R_{\Sigma}\right)-\frac{1}{Z\left(R_{\Sigma}\right)}\left(\frac{d R_{\Sigma}}{d T}\right)^{2}>0
$$

so that $\mathrm{T}$ is a timelike coordinate. From Eqs.(2.5), (2.10) and (2.11), it follows that

$$
\begin{array}{r}
R_{\Sigma}=(a f)_{\Sigma} \\
{\left[Z\left(R_{\Sigma}\right)-\frac{1}{Z\left(R_{\Sigma}\right)}\left(\frac{d R_{\Sigma}}{d T}\right)^{2}\right]^{\frac{1}{2}} d T=d t}
\end{array}
$$

Also, from Eqs.(2.8) and (2.9), the outward unit normals in $V^{-}$and $V^{+}$, respectively, are given by

$$
\begin{aligned}
& n_{\mu}^{-}=(0, a(t), 0,0), \\
& n_{\mu}^{+}=\left(-\dot{R}_{\Sigma}, \dot{T}, 0,0\right) .
\end{aligned}
$$


The components of extrinsic curvature $K_{i j}^{ \pm}$become

$$
\begin{aligned}
K_{00}^{-} & =0, \\
K_{22}^{-} & =\csc ^{2} \theta K_{33}^{-}=\left(f f^{\prime} a\right)_{\Sigma}, \\
K_{00}^{+} & =\left(\dot{R} \ddot{T}-\dot{T} \ddot{R}-\frac{Z}{2} \frac{d Z}{d R} \dot{T}^{3}+\frac{3}{2 Z} \frac{d Z}{d R} \dot{T} \dot{R}^{2}\right)_{\Sigma}, \\
K_{22}^{+} & =\csc ^{2} \theta K_{33}^{+}=(Z R \dot{T})_{\Sigma},
\end{aligned}
$$

where dot and prime mean differentiation with respect to $t$ and $\chi$ respectively. From Eq.(2.6), the continuity of extrinsic curvature gives

$$
\begin{aligned}
& K_{00}^{+}=0, \\
& K_{22}^{+}=K_{22}^{-} .
\end{aligned}
$$

Using Eqs.(2.17)-(2.22) along with Eqs.(2.4), (2.13) and (2.14), the junction conditions become

$$
\begin{array}{r}
\left(\dot{f}^{\prime}\right)_{\Sigma}=0, \\
M=\left(\frac{a f}{2}-\frac{\Lambda}{6}(a f)^{3}+\frac{Q^{2}}{2 a f}+\frac{a \dot{a}^{2}}{2} f^{3}-\frac{a}{2} f f^{\prime 2}\right)_{\Sigma} .
\end{array}
$$

Equations (2.13), (2.14), (2.23) and (2.24) provide the necessary and sufficient conditions for the smooth matching of the two regions over $\Sigma$.

\section{Solution of the Einstein Field Equations}

In this section, we solve the Einstein field equations with cosmological constant for the Friedmann models containing the charged perfect fluid as the source of gravitation. The Einstein field equations with cosmological constant are given by

$$
G_{\mu \nu}-\Lambda g_{\mu \nu}=\kappa\left(T_{\mu \nu}+T_{\mu \nu}^{(e m)}\right) .
$$

The energy-momentum tensor for perfect fluid is

$$
T_{\mu \nu}=(\rho+p) u_{\mu} u_{\nu}-p g_{\mu \nu},
$$

where $\rho$ is the energy density, $p$ is the pressure and $u_{\mu}=\delta_{\mu}^{0}$ is the four-vector velocity in co-moving coordinates. $T_{\mu \nu}^{(e m)}$ is the energy-momentum tensor for the electromagnetic field given by

$$
T_{\mu \nu}^{(e m)}=\frac{1}{4 \pi}\left(-g^{\delta \omega} F_{\mu \delta} F_{\nu \omega}+\frac{1}{4} g_{\mu \nu} F_{\delta \omega} F^{\delta \omega}\right) .
$$


With the help of Eqs.(3.2) and (3.3), Eq.(3.1) takes the form

$$
R_{\mu \nu}=8 \pi\left[(\rho+p) u_{\mu} u_{\nu}+\frac{1}{2}(p-\rho) g_{\mu \nu}+T_{\mu \nu}^{(e m)}-\frac{1}{2} g_{\mu \nu} T^{(e m)}\right]-\Lambda g_{\mu \nu} .
$$

Now we solve the Maxwell's field equations

$$
\begin{aligned}
F_{\mu \nu} & =\phi_{\nu, \mu}-\phi_{\mu, \nu}, \\
F^{\mu \nu} ; \nu & =4 \pi J^{\mu},
\end{aligned}
$$

where $\phi_{\mu}$ is the four potential and $J^{\mu}$ is the four current. Since the charge is at rest in this system, the magnetic field will be zero. Thus we can choose the four potential and four current as follows

$$
\phi_{\mu}=(\phi(t, r), 0,0,0), \quad J^{\mu}=\sigma u^{\mu}
$$

where $\sigma$ is charge density. Using Eqs.(3.5) and (3.7), the non-zero components of the field tensor are given as follows:

$$
F_{01}=-F_{10}=-\frac{\partial \phi}{\partial \chi} .
$$

Also, from Eqs.(3.6), (3.7) and (3.8), we have

$$
\begin{gathered}
\frac{\partial^{2} \phi}{\partial \chi^{2}}+2 \frac{f^{\prime}}{f}=4 \pi \sigma a^{2}, \\
a \frac{\partial^{2} \phi}{\partial \chi \partial t}+\dot{a} \frac{\partial \phi}{\partial \chi}=0 .
\end{gathered}
$$

Integration of Eq.(3.11) implies that

$$
\frac{\partial \phi}{\partial \chi}=\frac{1}{a f^{2}} q(\chi),
$$

where $q(\chi)=4 \pi \int_{0}^{\chi} \sigma a^{3} f^{2} d \chi$, is the total charge distribution in the interior spacetime. This amount of charge is the consequence of law of conservation of charge, i.e., $J_{; \mu}^{\mu}=0$. It is clear that Eq.(3.10) is identically satisfied by Eq.(3.11). The electromagnetic field intensity is given by

$$
E=\frac{q}{(a f)^{2}}
$$


Equations (3.11) and (3.12) yield

$$
\frac{\partial \phi}{\partial \chi}=a E .
$$

Using Eqs. (3.8) and (3.13), we get

$$
F_{01}=-F_{10}=-a E .
$$

The non-zero components of $T_{\mu \nu}^{(e m)}$ and its trace free form turn out to be

$$
\begin{aligned}
& T_{00}^{(e m)}=\frac{1}{8 \pi} E^{2}, \quad T_{11}^{(e m)}=-\frac{1}{8 \pi} E^{2} a^{2}, \quad T_{22}^{(e m)}=\frac{1}{8 \pi} E^{2}(a f)^{2}, \\
& T_{33}^{(e m)}=T_{22}^{(e m)} \sin ^{2} \theta, \quad T^{(e m)}=0 .
\end{aligned}
$$

When we use these values, the field equations (3.4) for the interior spacetime takes the form

$$
\begin{aligned}
& R_{00}=-3 \frac{\ddot{a}}{a}=4 \pi(\rho+3 p)+E^{2}-\Lambda \\
& R_{11}=-\frac{\ddot{a}}{a}-2 \frac{\dot{a}^{2}}{a^{2}}+\frac{2}{a^{2}} \frac{f^{\prime \prime}}{f}=4 \pi(p-\rho)+E^{2}-\Lambda \\
& R_{22}=-\frac{\ddot{a}}{a}-\left(\frac{\dot{a}}{a}\right)^{2}+\frac{1}{a^{2}}\left[\frac{f^{\prime \prime}}{f}+\left(\frac{f^{\prime}}{f}\right)^{2}-\frac{1}{f^{2}}\right]=4 \pi(p-\rho)-E^{2}-\Lambda(3.1 \\
& R_{33}=\sin ^{2} \theta R_{22}
\end{aligned}
$$

We would like to mention here that all the results are valid for $E^{2}<<\rho$ and hence for stiff matter $(\rho=p), E^{2}<<p$. Integrating Eq.(2.23) with respect to $t$, it follows that

$$
f^{\prime}=W,
$$

where $W=W(\chi)$ is an arbitrary function of $\chi$. The energy conservation equation

$$
T_{\mu ; \nu}^{\nu}=0
$$

for the perfect fluid with the interior metric shows that pressure is a function of $t$ only, i.e.,

$$
p=p(t) .
$$

Using the values of $f^{\prime}$ and $p$ from Eqs.(3.19) and (3.21) in Eqs.(3.15)-(3.17), it follows that

$$
2 \frac{\ddot{a}}{a}+\left(\frac{\dot{a}}{a}\right)^{2}+\frac{\left(1-W^{2}\right)}{(a f)^{2}}=\Lambda+E^{2}-8 \pi p(t) .
$$


We consider $p$ as a polynomial in $t$ as given by [15]

$$
p(t)=p_{c}\left(\frac{t}{T}\right)^{-s}
$$

where $T$ is the constant time introduced in the problem due to physical reason by re-scaling of $t, p_{c}$ and $s$ are positive constants. Further, for simplicity, we take $s=0$ so that

$$
p(t)=p_{c}
$$

Now Eq.(3.22) gives

$$
2 \frac{\ddot{a}}{a}+\left(\frac{\dot{a}}{a}\right)^{2}+\frac{\left(1-W^{2}\right)}{(a f)^{2}}=\Lambda+E^{2}-8 \pi p_{c} .
$$

For the static charges $E$ is taken as time independent [27], so integration of above equation with respect to $t$, yields

$$
\dot{a}^{2}=\frac{W^{2}-1}{f^{2}}+\left(\Lambda+E^{2}-8 \pi p_{c}\right) \frac{a^{2}}{3}+2 \frac{m}{a f^{3}},
$$

where $m=m(\chi)$ is an arbitrary function of $\chi$ and is related to the mass of the collapsing system. Substituting Eqs.(3.19), (3.26) into Eq.(3.15), we get

$$
m^{\prime}=\frac{2 E^{\prime} E}{3}(a f)^{3}+a^{3} f^{\prime} f^{2}\left[4 \pi\left(p_{c}+\rho\right)+2 E^{2}\right] .
$$

For physical reasons, we assume that $\left(p_{c}+\rho\right) \geqslant 0$. Integrating Eq.(3.27) with respect to $\chi$, we obtain

$$
m(\chi)=4 \pi a^{3} \int_{0}^{\chi}\left(\rho+p_{c}\right) f^{\prime} f^{2} d \chi+2 \int_{0}^{\chi} E^{2} f^{\prime} f^{2} d \chi+\frac{2}{3} a^{3} \int_{0}^{\chi} E^{\prime} E f^{3} d \chi+m_{0},
$$

where $m_{0}$ is taken equal to zero because of finite distribution of matter at the origin. The function $m(\chi)$ must be positive because $m(\chi)<0$ implies negative mass which is not physical. Using Eqs.(3.19) and (3.26) into the junction condition Eq.(2.24), it follows that

$$
M=\frac{Q^{2}}{2 a f}+m+\frac{1}{6}\left(\Lambda+E^{2}-8 \pi p_{c}\right)(a f)^{3} .
$$


The total energy $\tilde{M}(\chi, t)$ at time $t$ inside the hypersurface $\Sigma$ can be evaluated by using the definition of mass function with the contribution of electromagnetic field for the Friedmann model, which is given by

$$
\tilde{M}(\chi, t)=\frac{1}{2}(a f)\left(1+(\dot{a} f)^{2}-f^{\prime 2}\right)+\frac{q^{2}}{2 a f} .
$$

Replacing Eqs.(3.19) and (3.26) in Eq.(3.30), we obtain

$$
\tilde{M}(r, t)=m(r)+\left(\Lambda+E^{2}-8 \pi p_{c}\right) \frac{(a f)^{3}}{6}+\frac{q^{2}}{2 a f} .
$$

From Eqs.(3.29) and (3.31), it can be found that $\tilde{M}(r, t)={ }^{\Sigma} M$ if and only if $q=Q$. This result provides the necessary and sufficient conditions for the continuity of mass in the interior and exterior regions over boundary surface $\Sigma$.

Now we take $\left(\Lambda+E^{2}-8 \pi p_{c}\right)>0$ such that $E^{2}<<8 \pi p$ and assume that

$$
W(\chi)=1
$$

In order to obtain the analytic solutions in closed form, we use Eqs.(3.19), (3.26) and (3.32) so that

$$
(a f)=\left(\frac{6 m}{\Lambda+E^{2}-8 \pi p_{c}}\right)^{\frac{1}{3}} \sinh ^{\frac{2}{3}} \alpha(\chi, t)
$$

where

$$
\left.\alpha(\chi, t)=\frac{\sqrt{3\left(\Lambda+E^{2}-8 \pi p_{c}\right)}}{2}\left[t_{s}(\chi)-t\right)\right] .
$$

Here $t_{s}(\chi)$ is an arbitrary function of $\chi$ and is related to the time of formation of singularity.

\section{Apparent Horizons}

In this section, we discuss the formation of apparent horizons. The boundary of two trapped spheres whose outward normals are null is used to find the apparent horizons. Moreover, we discuss the the physical significance of apparent horizons i.e., area of apparent horizons, time difference between apparent horizons and singularity etc. For the interior spacetime, we find 
the boundary of two trapped spheres whose outward normals are null as follows:

$$
g^{\mu \nu}(a f)_{, \mu}(a f)_{, \nu}=(\dot{a} f)^{2}-\left(f^{\prime}\right)^{2}=0 .
$$

Using Eqs.(3.19) and (3.26) in this equation, we get

$$
\left(\Lambda+E^{2}-8 \pi p_{c}\right)(a f)^{3}-3(a f)+6 m=0 .
$$

When $\Lambda=8 \pi p_{c}-E^{2}$, we have $(a f)=2 m$. This is called Schwarzschild horizon. For $m=p_{c}=K=0$, we have $(a f)=\sqrt{\frac{3}{\Lambda}}$, which is called de-Sitter horizon. Equation (4.2) can have the following positive roots.

Case (i): For $3 m<\frac{1}{\sqrt{\left(\Lambda+E^{2}-8 \pi p_{c}\right)}}$, we obtain two horizons

$$
\begin{aligned}
(a f)_{c} & =\frac{2}{\sqrt{\left(\Lambda+E^{2}-8 \pi p_{c}\right)}} \cos \frac{\varphi}{3} \\
(a f)_{b} & =\frac{-1}{\sqrt{\left(\Lambda+8 \pi E^{2}-p_{c}\right)}}\left(\cos \frac{\varphi}{3}-\sqrt{3} \sin \frac{\varphi}{3}\right),
\end{aligned}
$$

where

$$
\cos \varphi=-3 m \sqrt{\left(\Lambda+E^{2}-8 \pi p_{c}\right)} .
$$

If we take $m=0$, it follows from Eqs.(4.3) and (4.4) that $(\text { af })_{c}=\sqrt{\frac{3}{\left(\Lambda+E^{2}-8 \pi p_{c}\right)}}$ and $(a f)_{b}=0$. (af $)_{c}$ and $(a f)_{b}$ are called cosmological horizon and black hole horizons respectively. For $m \neq 0$ and $\Lambda \neq 8 \pi p_{c}-E^{2}$, (af $)_{c}$ and $(a f)_{b}$ can be generalized [28] respectively.

Case (ii): For $3 m=\frac{1}{\sqrt{\left(\Lambda+E^{2}-8 \pi p_{c}\right)}}$, there is only one positive root which corresponds to a single horizon i.e.,

$$
(a f)_{c}=(a f)_{b}=\frac{1}{\sqrt{\left(\Lambda+E^{2}-8 \pi p_{c}\right)}}=(a f)_{c b}
$$

This shows that both horizons coincide. The range for the cosmological and black hole horizon can be written as follows

$$
0 \leq(a f)_{b} \leq \frac{1}{\sqrt{\left(\Lambda+E^{2}-8 \pi p_{c}\right)}} \leq(a f)_{c} \leq \sqrt{\frac{3}{\left(\Lambda+E^{2}-8 \pi p_{c}\right)}}
$$


The black hole horizon has its largest proper area $4 \pi(a f)^{2}=\frac{4 \pi}{\left(\Lambda+E^{2}-8 \pi p_{c}\right)}$ and cosmological horizon has its area between $\frac{4 \pi}{\left(\Lambda+E^{2}-8 \pi p_{c}\right)}$ and $\frac{12 \pi}{\left(\Lambda+E^{2}-8 \pi p_{c}\right)}$.

Case (iii): For $3 m>\frac{1}{\sqrt{\left(\Lambda+E^{2}-8 \pi p_{c}\right)}}$, there are no positive roots and consequently there are no apparent horizons.

We now calculate the time of formation of the apparent horizon using Eqs.(3.33) and (4.2)

$$
t_{n}=t_{s}-\frac{2}{\left.\sqrt{3\left(\Lambda+E^{2}-8 \pi p_{c}\right.}\right)} \sinh ^{-1}\left(\frac{(a f)_{n}}{2 m}-1\right)^{\frac{1}{2}}, \quad(n=1,2) .
$$

This implies that

$$
\frac{(a f)_{n}}{2 m}=\cosh ^{2} \alpha_{n}
$$

where $\left.\alpha_{n}(r, \chi)=\frac{\sqrt{3\left(\Lambda+E^{2}-8 \pi p_{c}\right)}}{2}\left[t_{s}(\chi)-t_{n}\right)\right]$. Equations (3.33) and (4.6) give $(a f)_{c} \geq(a f)_{b}$ and $t_{b} \geq t_{c}$ respectively. The inequality $t_{b} \geq t_{c}$ indicates that the cosmological horizon forms earlier than the black hole horizon. This condition confirms the formation of black hole.

The time difference between the formation of cosmological horizon and singularity and the formation of black hole horizon and singularity can be found as follows. Using Eqs.(4.3)-(4.5), it follows that

$$
\begin{aligned}
& \frac{d\left(\frac{(a f)_{c}}{2 m}\right)}{d m}=\frac{1}{m}\left(-\frac{\sin \frac{\varphi}{3}}{\sin \varphi}+\frac{3 \cos \frac{\varphi}{3}}{\cos \varphi}\right)<0, \\
& \frac{d\left(\frac{(a f)_{b}}{2 m}\right)}{d m}=\frac{1}{m}\left(-\frac{\sin \frac{(\varphi+4 \pi)}{3}}{\sin \varphi}+\frac{3 \cos \frac{(\varphi+4 \pi)}{3}}{\cos \varphi}\right)>0 .
\end{aligned}
$$

The time difference between the formation of singularity and apparent horizons is

$$
\tau_{n}=t_{s}-t_{n}
$$

It follows from Eq.(4.9) that

$$
\frac{d \tau_{n}}{d\left(\frac{Y_{n}}{2 m}\right)}=\frac{1}{\sinh \alpha_{n} \cosh \alpha_{n} \sqrt{3\left(\Lambda+E^{2}-8 \pi p_{c}\right)}} .
$$


Using Eqs.(4.10) and (4.13), we get

$$
\begin{array}{r}
\frac{d \tau_{1}}{d m}=\frac{d \tau_{1}}{d\left(\frac{(a f)_{c}}{2 m}\right)} \frac{d\left(\frac{(a f)_{c}}{2 m}\right)}{d m}=\frac{1}{m \sqrt{3\left(\Lambda+E^{2}-8 \pi p_{c}\right)} \sinh \alpha_{1} \cosh \alpha_{1}} \\
\times\left(-\frac{\sin \frac{\varphi}{3}}{\sin \varphi}+\frac{3 \cos \frac{\varphi}{3}}{\cos \varphi}\right)<0 .
\end{array}
$$

This means that time interval between the formation of cosmological horizon and singularity is decreased with the increase of mass. Similarly, from Eqs.4.11) and(4.13), we get

$$
\begin{aligned}
& \frac{d \tau_{2}}{d m}=\frac{1}{m \sqrt{3\left(\Lambda+E^{2}-8 \pi p_{c}\right)} \sinh \alpha_{2} \cosh \alpha_{2}} \\
& \times\left(-\frac{\sin \frac{(\varphi+4 \pi)}{3}}{\sin \varphi}+\frac{3 \cos \frac{(\varphi+4 \pi)}{3}}{\cos \varphi}\right)>0 .
\end{aligned}
$$

This indicates that time difference between the formation of black hole horizon and singularity is increased with the increase of mass.

\section{$5 \quad$ Singularity Analysis}

The Riemann tensor is used to determine whether a singularity is essential or removable. If the curvature becomes infinite at certain point, then the singularity will be essential otherwise removable. Many scalars can be constructed from the Riemann tensor but symmetry assumption can be used to find only a finite number of independent scalars. Some of these are

$$
R_{1}=R=g^{a b} R_{a b}, \quad R_{2}=R_{a b} R^{a b}, \quad R_{3}=R_{a b c d} R^{a b c d}, \quad R_{4}=R_{c d}^{a b} R_{a b}^{c d} .
$$

Here, we give the analysis for the first invariant commonly known as the Ricci scalar. For the Friedmann model, it is given as

$$
R=\frac{-3 a \ddot{a} f^{2}+2 f^{\prime \prime} f-3 \dot{a}^{2} f-1+f^{2}}{a^{2} f} .
$$

By definition $a>0$ and $\frac{\dot{a}}{a}>0$ [29], it follows that curves of $a(t)$ versus $t$ must be concave downward and must reach $a(t)=0$ at some finite time in the past. Let us recall this time $t=0$ at which $R=\infty$. In cosmology, 
extrapolation of the universe expansion backwards in time yields an infinite density at finite past. Also, if the strong energy condition [1] is satisfied, i.e., $\rho+p \geq 0$ and $(\rho+3 p) \geq 0$ then $a=0$ at $t=0$ which implies the divergence of scalar curvature polynomial where $\rho \rightarrow \infty$. This is spacelike singularity usually called big bang singularity or initial singularity [30].

\section{Summary and Conclusion}

In this paper, we have analyzed the charged perfect fluid collapse with positive cosmological constant in the Friedmann models. For this purpose, we have found junction conditions between the Friedmann models and the Reissner-Nordström de-Sitter spacetime. The junction conditions provide the gate way for the exact solution of the field equations with interior spacetime (Friedmann models). The solution of the field equations helps to discuss the dynamics of the collapsing system as follows:

The acceleration parameter $\ddot{a} / a$, given by Eq.(2.14), will be zero, positive or negative for $4 \pi(\rho+3 p)+E^{2}-\Lambda=0,4 \pi(\rho+3 p)+E^{2}-\Lambda<0$ or $4 \pi(\rho+3 p)+E^{2}-\Lambda>0$ respectively. The variation of the scale factor $a(t)$ helps to describe the different stages of matter in the Friedmann models of the universe [31. If the scale factor $a(t)$ is decreasing, i.e., $\dot{a}(t)<0$ there will be collapsing (contracting) phase. For increasing scale factor i.e., $\dot{a}(t)>0$ we have the expanding phase while the point where $\dot{a}(t)=0$ corresponds to bounce point. Consequently, the Hubble parameter will be $H<0, H>0$ and $H=0$ for collapsing, expanding and bouncing phases respectively. Also, we can conclude the following:

- The Newtonian force and acceleration of matter have the same value over the hypersurface $\Sigma$, i.e., $\left(-\frac{m}{(a f)^{2}}+\left(\Lambda+E^{2}-8 \pi p_{c}\right) \frac{(a f)}{3}\right)_{\Sigma}$ (see [23] for detail). In this case, the repulsive force can only be generated if $\Lambda>\left(8 \pi p_{c}-E^{2}\right)$ such that $8 \pi p_{c}>>E^{2}$ over the entire range of the collapsing sphere. In the case of charged perfect fluid collapse with Tolman-Bondi spacetime [23] there is no restriction on matter and electromagnetic field then the results are valid only for $\Lambda>\left(8 \pi p_{c}-E^{2}\right)$ such that $8 \pi p_{c}>E^{2}$. It is clear that in the first case the cosmological constant attains higher value than the later case. Thus the cosmological constant plays an effective role to slow down the collapse in the present case than previous one. In other words, isotropy and homogeneity of 
matter causes to introduce resistance against collapse in the presence of charge.

- Since the cosmological constant $\Lambda$ is affected by pressure and electromagnetic field, we can say that electromagnetic field reduces the effects of $\Lambda$ as compared to perfect fluid case by putting the restriction on $\Lambda$. Hence electromagnetic field increases the gravitational collapse as it decreases the repulsive force produced by $\Lambda$.

- Two physical horizons (cosmological and black hole horizons) are found whose area is decreased by cosmological constant and electromagnetic field. It follows from Eq.(4.8) that both horizons form earlier than singularity, so singularity is covered (back hole) and $\mathrm{CCH}$ seems to be valid in this case.

- Time difference between the formation of apparent horizon and singularity is decreased by electromagnetic field. Thus we can say that singularity must form earlier than the apparent horizons. Hence electromagnetic field favors the formation of naked singularity. But such situation can never occur because electromagnetic field does not play the dominant role in this case.

- It is found that the time difference between the formation of cosmological (black) horizon and singularity is decreasing (increasing) function of mass of the collapsing system.

\section{Acknowledgment}

We would like to thank the Higher Education Commission, Islamabad, Pakistan for its financial support through the Indigenous Ph.D. 5000 Fellowship Program Batch-IV.

\section{References}

[1] Hawking, S.W. and Ellis, G.F.R.: The Large Scale Structure of Spacetime (Cambridge University Press, Cambridge 1975).

[2] Penrose, R.: Riv. Nuovo Cimento: 1(1969)252. 
[3] Virbhadra, K.S., Narasimha, D. and Chitre, S.M.: Astron. Astrophys. $337(1998) 1$.

[4] Virbhadra, K.S., and Ellis, G.F.R.: Phys. Rev. D62(2000)084003.

[5] Virbhadra, K.S., and Ellis, G.F.R.: Phys. Rev. D65(2002)103004.

[6] Virbhadra, K.S.: D79(2009)083004.

[7] Oppenheimer, J.R. and Snyder, H.: Phys. Rev. 56(1939)455.

[8] Eardley, D.M. and Smarr, L.: Phys. Rev. D19(1979)2239.

[9] Misner, C.W. and Sharp, D.: Phys. Rev. 136(1964)b571.

[10] Vaidya, P.C.: Proc. Indian Acad. Sci. A33(1951)264.

[11] Santos, N.O.: Phys. Lett. A106(1984)296.

[12] Markovic, D. and Shapiro, S.L.: Phys. Rev. D61(2000)084029.

[13] Lake, K.: Phys. Rev. D62(2000)027301.

[14] Sharif, M. and Ahmad, Z.: Mod. Phys. Lett. A22(2007)1493.

[15] Sharif, M. and Ahmad, Z.: Mod. Phys. Lett. A22(2007)2947.

[16] Sharif, M. and Ahmad, Z.: J. Korean Phys. Society 52(2008)980.

[17] Sharif, M. and Ahmad, Z.: Acta Phys. Polonica B39(2008)1337.

[18] Sharif, M. and Ahmad, Z.: Int. J. Mod. Phys. A23(2008)181.

[19] Sharif, M. and Iqbal, K.: Mod. Phys. Lett. A24(2009)1533.

[20] Thirukkanesh, S. and Maharaj, S. D.: Math. Meth. Appl. Sci. 32(2009)684.

[21] Sharma, R., Mukharjee, S. and Maharaj, S.D.: Gen. Relativ. Grav. 33(2001)999.

[22] Nath, S., Debnath, U. and Chakraborty, S.: Astrophys. Space Sci. 313(2008)431. 
[23] Sharif, M. and Abbas, G.: Mod. Phys. Lett. A24(2009)2551.

[24] Dozmorov, I.M. and Semenov, V.P.: Russian Phy. J. 16(1973)1064.

[25] Tsagas C.G.: Class. Quantum Grav. 22(2005)393.

[26] Darmois, G.: Memorial des Sciences Mathematiques (Gautheir-Villars, Paris, 1927) Fasc. 25.

[27] Bakshi, A.V. and Bakshi, U.A.: Field Theory (Technical Publication Pune, India 2007).

[28] Hayward, S.A., Shiromizu, T. and Nakao, K.: Phys. Rev. D49(1994)5080.

[29] Weinberg, S.: Gravitation and Cosmology (John Wiley and Sons, Singapore, 2005).

[30] Harada, T.: Advanced Studies in Pure Math. 55(2009)15.

[31] Nozari, K. and Sadatian, S.D.: Phys. Lett. B676(2009)1. 